\title{
Front Matter: Volume 10914
}

, "Front Matter: Volume 10914," Proc. SPIE 10914, Optical Components and Materials XVI, 1091401 (10 May 2019); doi: 10.1117/12.2531134

SPIE. Event: SPIE OPTO, 2019, San Francisco, California, United States 


\section{PROCEEDINGS OF SPIE}

\section{Optical Components and Materials XVI}

Shibin Jiang

Michel J. F. Digonnet

Editors

4-6 February 2019

San Francisco, California, United States

Sponsored and Published by

SPIE 
The papers in this volume were part of the technical conference cited on the cover and title page. Papers were selected and subject to review by the editors and conference program committee. Some conference presentations may not be available for publication. Additional papers and presentation recordings may be available online in the SPIE Digital Library at SPIEDigitalLibrary.org.

The papers reflect the work and thoughts of the authors and are published herein as submitted. The publisher is not responsible for the validity of the information or for any outcomes resulting from reliance thereon.

Please use the following format to cite material from these proceedings:

Author(s), "Title of Paper," in Optical Components and Materials XVI, edited by Shibin Jiang, Michel J. F. Digonnet, Proceedings of SPIE Vol. 10914 (SPIE, Bellingham, WA, 2019) Seven-digit Article CID Number.

ISSN: 0277-786X

ISSN: 1996-756X (electronic)

ISBN: 9781510624702

ISBN: 9781510624719 (electronic)

Published by

SPIE

P.O. Box 10, Bellingham, Washington 98227-0010 USA

Telephone +1 3606763290 (Pacific Time) · Fax +1 3606471445

SPIE.org

Copyright (C) 2019, Society of Photo-Optical Instrumentation Engineers.

Copying of material in this book for internal or personal use, or for the internal or personal use of specific clients, beyond the fair use provisions granted by the U.S. Copyright Law is authorized by SPIE subject to payment of copying fees. The Transactional Reporting Service base fee for this volume is $\$ 18.00$ per article (or portion thereof), which should be paid directly to the Copyright Clearance Center (CCC), 222 Rosewood Drive, Danvers, MA 01923. Payment may also be made electronically through CCC Online at copyright.com. Other copying for republication, resale, advertising or promotion, or any form of systematic or multiple reproduction of any material in this book is prohibited except with permission in writing from the publisher. The CCC fee code is 0277$786 \mathrm{X} / 19 / \$ 18.00$.

Printed in the United States of America by Curran Associates, Inc., under license from SPIE.

Publication of record for individual papers is online in the SPIE Digital Library.

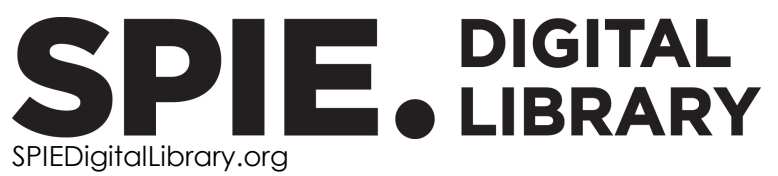

Paper Numbering: Proceedings of SPIE follow an e-First publication model. A unique citation identifier (CID) number is assigned to each article at the time of publication. Utilization of CIDs allows articles to be fully citable as soon as they are published online, and connects the same identifier to all online and print versions of the publication. SPIE uses a seven-digit CID article numbering system structured as follows:

- The first five digits correspond to the SPIE volume number.

- The last two digits indicate publication order within the volume using a Base 36 numbering system employing both numerals and letters. These two-number sets start with $00,01,02,03,04$, 05, 06, 07, 08, 09, 0A, OB ... 0Z, followed by 10-1Z, 20-2Z, etc. The CID Number appears on each page of the manuscript. 


\section{Contents}

$\begin{array}{ll}\text { vii } & \text { Authors } \\ \text { xi } & \text { Conference Committee }\end{array}$

RARE-EARTH DOPED MATERIALS I

$1091403 \quad 1.5 \mu \mathrm{m}$ persistent luminescence of $\mathrm{Er}^{3+}$ in $\mathrm{Gd}_{3} \mathrm{Al}_{5-\mathrm{x}} \mathrm{Ga}_{\mathrm{x}} \mathrm{O}_{12}$ (GAGG) garnets via persistent energy transfer (Invited Paper) [10914-2]

1091405 High-power fiber laser materials: influence of fabrication methods and codopants on optical properties [10914-4]

$1091406 \quad 405$-nm pumped $\mathrm{Ce}^{3+}$-doped silica fiber for broadband fluorescence from cyan to red [10914-5]

\section{METAMATERIALS}

1091409 Nanoimprinted nanocomposite membrane-type metamaterials [10914-8]

10914 OA Arsenic selenide dielectric metasurfaces [10914-9]

\section{SILICON PHOTONICS}

10914 OB Si photonics using micron-size waveguides (Invited Paper) [10914-10]

SENSORS

10914 OF Substrate requirements to enable durability and accuracy in structured-light-based 3D sensing [10914-14]

\section{GRATINGS}

$109140 \mathrm{~J}$ Low-loss and robust DWDM Echelle grating (de-)multiplexers in SOI technology [10914-18]

10914 OK Bloch surface wave excitation using a maximum length sequence grating structure [10914-19] 
10914 OL Ytterbium-doped nanostructured core silica fiber with built-in Bragg grating for laser applications [10914-20]

\section{NANOMATERIALS}

10914 OR Analysis of upconversion nanoparticles as an active medium for upconversion light sources [10914-26]

10914 OS Phase-dependent emission of $\mathrm{KLaF}_{4}: \mathrm{Nd}^{3+}$ nanocrystals in oxyfluoride glass-ceramics [10914-27]

10914 OT Site symmetry and host sensitization-dependence of Eu ${ }^{3+}$ real-time luminescence in tin dioxide nanoparticles (Invited Paper) [10914-28]

OPTICAL GLASSES, FIBERS, AND WAVEGUIDES

$109140 \mathrm{~V} \quad$ Optical glass: refractive index homogeneity from small to large parts - an overview [10914-30]

$109140 X \quad$ Fabrication of high optical quality Ge-As-Se glasses for the development of low-loss microstructured optical fibers [10914-32]

10914 OY Novel approach for high-performance optical fibers: multiple-doped silica powders with plasma-enhanced processes [10914-33]

$109140 Z$ Optical properties and long-term stability of unclad single crystal sapphire fiber in harsh environments [10914-34]

$1091410 \quad$ Flexible waveguides with amorphous photonic materials [10914-35]

\section{RARE-EARTH DOPED MATERIALS II}

$1091411 \quad \mathrm{SiO}_{2}-\mathrm{SnO}_{2}$ transparent glass-ceramics activated by rare earth ions (Invited Paper) [10914-36]

1091412 Small short-wavelength optical isolator using Tb ${ }^{3+}$-rich magneto-optical glass [10914-37]

\section{DETECTORS}

$1091415 \quad$ Linearity characterization of high performance SWIR photodetectors from various materials [10914-40]

1091416 Advances on photoconductive InAs/GaSb type-II superlattice long-wavelength infrared detectors for high operating temperature [10914-41]

iv 
1091417 Metal semiconductor metal photodiodes based on all-epitaxial Ge-on-insulator-on- Si(111), grown by molecular beam epitaxy [10914-42]

$1091418 \quad$ Ultrafast response vertical phototransistors based on hybrid perovskite [10914-43]

1091419 Solution-possessed vertical photodetectors based on composition-dependent cesium lead halide $\left(\mathrm{CsPbX}_{3}, \mathrm{X}=\mathrm{Cl}, \mathrm{Br}\right.$, and I) perovskite quantum dots [10914-44]

10914 1A Metal-semiconductor-metal photodetectors on a GeSn-on-insulator platform [10914-45]

\section{DEVELOPMENT OF OPTICAL COMPONENTS}

10914 1B Super broadband achromatic lenses extending from the visible to the mid-infrared [10914-46]

$10914 \mathrm{lE} \quad$ Automated sprue removal from injection moulded micro-optics with ultrasonic cutting [10914-50]

$10914 \mathrm{IF} \quad$ Optics with diamond-like-carbon overcoat (DOC) provide improved optical performance over traditional DLC films and better cleanability than standard PVD coatings [10914-51]

\section{POSTER SESSION}

$109141 \mathrm{H} \quad$ Compositional dependence of the emission color of sodium borate glasses embedded with inorganic ions under diode laser excitation [10914-53]

$1091411 \quad$ First-principle calculations of Debye temperature of optoelectronic LiGaS 2 and LiGaSe2 semiconductors under different pressures [10914-54]

$10914 \mathrm{lJ} \quad$ Numerical investigation on local confinement of infrared light in chalcogenide transversely disordered optical fibers [10914-57]

$109141 \mathrm{~K} \quad$ Diamond gratings used for high-power laser system [10914-58]

$109141 \mathrm{M} \quad$ Structure optimization of $\mathrm{KTa}_{1-x} \mathrm{Nb}_{x} \mathrm{O}_{3}$ varifocal lens [10914-60]

$109141 \mathrm{~N} \quad$ Monitoring the purification of tobacco smoke in air assisted by ZnO nanowires and using MEMS-FTIR spectrometer for online continuous analysis of volatile organic compounds (VOCs) [10914-61]

1091410 Tailoring bandgap transmission spectra of new neodymium-doped tellurite all-solid photonic bandgap fibers with double cladding layers [10914-62]

10914 IR Single-mode large-mode-area Er-Yb fiber [10914-65]

10914 1S 3D printing optical devices based on silicone optical technology (SOT) and its application on analytical chemistry [10914-66] 
$109141 \mathrm{U} \quad$ Optical properties of partially hydrogenated graphene using first-principle calculations [10914-70]

$109141 \mathrm{~V}$ Coating geometry measurement of specialty fiber with dark-field illumination technique [10914-71]

10914 IW Diffractive optical elements investigation in the phase domain [10914-72]

$109141 X \quad$ Novel optical gas sensor based on photonic crystal fiber [10914-73]

1091412 Design of long-range hybrid plasmonic waveguides [10914-77]

1091420 Multi-wavelength erbium-doped fiber ring lasers based on an optical fiber tip interferometer [10914-78]

1091423 The sealed, the athermaled, and the rugged: the wild west of modified opto-mechanical design [10914-82]

$1091424 \quad$ High-efficiency Ge-on-Si SPADs for short-wave infrared [10914-84]

1091425 Temperature cross-sensitivity compensation in liquid level sensor using Mach-Zehnder interferometers [10914-56] 


\section{Authors}

Numbers in the index correspond to the last two digits of the seven-digit citation identifier (CID) article numbering system used in Proceedings of SPIE. The first five digits reflect the volume number. Base 36 numbering is employed for the last two digits and indicates the order of articles within the volume. Numbers start with 00, 01, 02, 03, 04, 05, 06, 07, 08, 09, OA, OB...0Z, followed by 10-12, 20-2Z, etc.

\author{
Aalto, Timo, OB \\ Abramov, Alexey N., 1R \\ Ahmed, Kaleem, 12 \\ Aleshkina, Svetlana S., IR \\ Amrithanath, Abhishek K., 20 \\ André, Paulo S. B., 25 \\ Antunes, Paulo F. C., 25 \\ Anuszkiewicz, Alicja, OL \\ Aras, Mehmet Sirin, 10 \\ Armellini, Cristina, 11 \\ Bacon, Jim, IF \\ Baeva, M., OY \\ Baierl, H., OY \\ Balda, Rolindes, OS, OT \\ Bartelt, $\mathrm{H}$, OY \\ Bekele, Robel Y., OA \\ Bera, S., OZ \\ Berger, M., 1E \\ Berneschi, Simone, 11 \\ Bhardwaj, Priyanka, 12 \\ Bhat, Srivathsa, OB \\ Bobkov, Konstantin K., IR \\ Bond, David, 15 \\ Boulard, Brigitte, 11 \\ Bourouina, Tarik, $1 \mathrm{~N}$ \\ Brecher, C., 1E \\ Brilland, Laurent, OX \\ Bubnov, Mikhail M., IR \\ Buczynski, Ryszard, OL \\ Buller, Gerald S., 24 \\ Buric, M., $\mathrm{OZ}$ \\ Cabral, A. A., OS \\ Caillaud, Céline, OX \\ Cao, Mingxuan, 19 \\ Carpentiero, Alessandro, 11 \\ Cascales, Concepción, OT \\ Chahal, Radwan, OX \\ Chandra, S., 11 \\ Che, Yongli, 19 \\ Chen, Zhiliang, 18, 19 \\ Cherchi, Matteo, OB \\ Chiappini, Andrea, 11 \\ Chiasera, Alessandro, 11 \\ Chichkov, Nikolay B., 06 \\ Chorpening, B., OZ \\ Clabeau, Anthony, OA \\ Daumer, Volker, 16 \\ Davis, Gregg, $1 \mathrm{~F}$
}

Dellith, J., OY

Dhawan, Anuj, 12

Dianov, Evgeny M., 06

Díaz, Camilo A. R., 25

Dorosz, Dominik, 11

Dumas, Derek C. S., 24

Durán, A., OS

Eberhardt, R., 05

Edwards, Vernessa M., $1 \mathrm{H}$

Eisenblätter, L., OJ

Erdil, Mertcan, 10

Erfan, Mazen, $1 \mathrm{~N}$

Fan, Shuwei, $1 \mathrm{~K}$

Fathy, Alaa, iN

Fedotov, A., OR

Fernández, Joaquín, OS, OT

Ferrari, Maurizio, 11

Filipkowski, Adam, OL

Foest, R., OY

Franczyk, Marcin, OL

Frantz, Jesse A., OA

Frizera, Anselmo, 25

Fujita, Naoki, 12

García-Revilla, Sara, OT

Gates, James, 11

Ghosh, Rajib Ratan, 12

Gnambodoe-Capo-Chichi, Martine, IN

Gorni, G., OS

Gumenyuk, Regina, 06, OR

Guo Xin, Tina, IA

Guryanov, Aleksey N., IR

Haapalinna, Atte, $\mathrm{OB}$

Haarlammert, N., 05

Hameed, Mohamed Farhat O., IX

Hanninen, Adam, 1B

Harjanne, Mikko, OB

Harris, Lori, 15

Hedges, Alan R., 1F

Heikal, Ahmed M., IX

Hein, S., 05

Hemmati, Hafez, 09

Hempel, F., OY

Hermerschmidt, Andreas, IW

Himelinski, Stan, $1 \mathrm{~F}$

Hoeren, M., IE

Hokkanen, Ari, OB

Holmberg, Heikki, OB

Hupel, C., 05 
lacob, Erica, 11

Imai, T., IM

Ischia, Gloria, 11

Janaszek, Andrzej, 16

Jedamzik, Ralf, OV

Jędrzejewski, Kazimierz, OL

Jin, Lufan, 18, 19

Jones, Andrew, $1 \mathrm{H}$

Jung, Chang Hyun, IV

Jung, Derek Minwoo, $1 \mathrm{~V}$

Jureńczyk, Jarosław, 16

Kapulainen, Markku, OB

Karnick, D., OJ

Kasztelanic, Rafał, OL

Kawamura, S., $1 \mathrm{M}$

Khiangte, K. R., 17

Khudyakov, Maxim M., IR

Kim, Dong Cheon, IW

Kim, Jaesun, $1 \mathrm{~V}$

Kim, Myun-Sik, IW

Kim, Young Jai, $1 \mathrm{~N}$

Kimchi, Joe, 15

Kirdoda, Jarosław, 24

Kocaman, Serdar, 10

Kochergina, Tatyana A., IR

Kohen, David, IA

Kong, Byung Joo, IV

Köpp, D., OY

Krishnaswamy, Sridhar, 20

Kuhn, S., 05

Kühner, T., OJ

Kumar, V., 11, $1 \mathrm{U}$

Kuzmenko, Kateryna, 24

Laha, A., 17

Leal, Arnaldo, Jr., 25

Lee, Junho, IV

Lee, Kwang Hong, IA

Lehtimäki, Lauri, OB

Lei, Jih-Fen, 15

Le Pivert, Marie, $1 \mathrm{~N}$

Leprince-Wang, Yamin, IN

Li, Qingyan, 18, 19

Li, Tengteng, 18

Li, Wei, $1 \mathrm{~A}$

Li, Yifan, 18, 19

Liggins, Kristopher, $1 \mathrm{H}$ Likhachev, Mikhail E., IR

Lin, Yiding, $1 \mathrm{~A}$

Lipatov, Denis S., $1 \mathrm{R}$

Lisowska, Jolanta, OL

Litchinitser, Natalia, OA

Liu, B., OZ

Lobanov, Aleksey S., IR

Lukowiak, Anna, 11

Magnusson, Robert, 09

Mahapatra, S., 17

Malasuk, Chacriya, is

Margetis, Joe, $1 \mathrm{~A}$

Markowski, Konrad, OL
Massella, Damiano, 11

McClain, Collin C., OA

Melkumov, Mikhail A., 06

Meneghetti, Marcello, OX

Methling, R., OY

Micheli, Victor, 11

Millar, Ross W., 24

Moon, Dae Seung, $1 \mathrm{~V}$

Morita, Kinichi, 1S

Müller, Raphael, 16

Müller, T., 1E

Murata, Daisuke, 03

Myers, Jason D., OA

Nakakubo, Keisuke, is

Nakatani, Asuka, $1 \mathrm{~J}$

Nguyen, Vinh, OA

Niemasz, Jasmin, 16

Niemi, T., OR

Nishiharaguchi, Nobuhiko, 10

Nold, J., 05

Nunzi Conti, Gualtiero, 11

Obayya, S.S. A., $1 \mathrm{X}$

Ohishi, Yasutake, $1 \mathrm{~J}, 10$

Ohodnicki, P., $0 Z$

Oki, Yuji, is

Orlovskaya, E. O., OR

Orlovskii, Yu. V., OR

Osten, H. J., 17

Osuch, Tomasz, OL

Ouh, Chihwan, IV

Owens, Ashley, $1 \mathrm{H}$

Park, Gaye, IV

Pascual, M. J., OS

Paul, Douglas J., 24

Pause, Philip, 23

Pelli, Stefano, 11

Petzold, Uwe, OV

Pokharia, R. S., 17

Pominova, D., OR

Pontes, Maria J., 25

Potma, Eric O., 1B

Poudel, Khem N., OK

Prudenzano, Francesco, 11

Pysz, Dariusz, OL

Rafailov, Edik U., 06

Ramponi, Roberta, 11

Rathore, J. S., 17

Reddy, B. Rami, $1 \mathrm{H}$

Rehm, Robert, 16

Reichel, $\mathrm{V}$., OY

Ribeiro, Moisés R. N., 25

Righini, Giancarlo C., 11

Robertson, William M., OK

Rossi, Barbara, 11

Rumyantsev, Andrey, 15

Saber, Ahmed, $1 \mathrm{X}$

Sabry, Yasser M., iN

Sanghera, Jasbinder S., OA

Santosh, R., IU 
Sarihan, Murat Can, 10

Sato, Fumio, 12

Saver, S., $1 \mathrm{E}$

Sazio, Pier-John, 11

Scharf, Toralf, IW

Scheffel, A., OY

Schmidt, J., 17

Schneider, M., OJ

Schreiber, Horst, OF

Schreiber, T., 05

Shin, Woojin, IV

Singh, Yadvendra, 11, $1 \mathrm{U}$

Son, Bong Kwon, 1A

Song, Kai, 15

Song, Zhiqiang, $1 \mathrm{~K}$

Speranza, Giorgio, 11

Stefaniuk, Tomasz, OL

Stutzki, F., 05

Subramanian, Senthil, 12

Sun, Fei, OB

Suzaki, Kohei, 10

Suzuki, Futoshi, 12

Suzuki, Takenobu, $1 \mathrm{~J}, 10$

Symeonidis, Michail, IW

Tan, Chuan Seng, 1A

Tanabe, Setsuhisa, 03

Thomas, Arun, 12

Tolle, John, IA

Tran, Lam Thi Ngoc, 11

Trautvetter, T., OY

Troles, Johann, OX

Trono, Cosimo, 11

Tuan, Tong Hoang, $1 \mathrm{~J}, 10$

Tünnermann, A., 05

Ueda, Jumpei, 03

Vaccari, Alessandro, 11

Varas, Stefano, 11

Vechkanov, Nikolay N., IR

Vehmas, Tapani, OB

Velázquez, J. J., OS

Vines, Peter, 24

Wang, Hong, $1 \mathrm{~A}$

Wang, Hongxing, $1 \mathrm{~K}$

Weber, M., OJ

Wei, Heming, 20

Wondraczek, K., OY

Wondraczek, L., OY

Wong, Chee Wei, 10

Wuenschell, J., $\mathrm{OZ}$

$\mathrm{XU}$, Jian, 03

Yadav, Amit, 06

Yanik, Cenk, 10

Yao, Jianquan, 18, 19

Yashkov, Mikhail V., 06

Yilmaz, Yildirim Batuhan, 10

Yoshioka, Hiroaki, $1 \mathrm{~S}$

Yu, Yu, 18, 19

Yuan, Henry, 15

Zhang, Lin, 1A
Zhang, Y., OJ

Zhang, Yating, 18, 19

Zherebtsov, Evgeny, 06

Zonta, Daniele, 11

Zontar, D., 1E

Zur, Lidia, 11 
Proc. of SPIE Vol. 10914 1091401-10 Downloaded From: https://www.spiedigitallibrary.org/conference-proceedings-of-spie on 26 Apr 2023
Terms of Use: https://www.spiedigitallibrary.org/terms-of-use 


\section{Conference Committee}

Symposium Chairs

Connie J. Chang-Hasnain, University of California, Berkeley (United States)

Graham T. Reed, Optoelectronics Research Center (United Kingdom)

Symposium Co-chairs

Sailing He, KTH Royal Institute of Technology (Sweden) and Zhejiang University (China)

Yasuhiro Koike, Keio University (Japan)

Program Track Chairs

James G. Grote, Photonics Consultant (United States)

Shibin Jiang, AdValue Photonics, Inc. (United States)

Conference Chairs

Shibin Jiang, AdValue Photonics, Inc. (United States)

Michel J. F. Digonnet, Stanford University (United States)

Conference Program Committee

Jean-Luc Adam, Université Rennes 1 (France)

Joel Bagwell, Edmund Optics Inc. (United States)

Rolindes Balda, Universidad del País Vasco (Spain)

Robert P. Dahlgren, NASA Ames Research Center (United States)

Angel Flores, Air Force Research Laboratory (United States)

Jesse A. Frantz, U.S. Naval Research Laboratory (United States)

Leonid B. Glebov, CREOL, The College of Optics and Photonics, University of Central Florida (United States)

Seppo K. Honkanen, University of Eastern Finland (Finland)

Jacques Lucas, Université de Rennes 1 (France)

Yasutake Ohishi, Toyota Technological Institute (Japan)

Aydogan Ozcan, University of California, Los Angeles (United States)

Giancarlo C. Righini, Istituto di Fisica Applicata "Nello Carrara" (Italy)

Setsuhisa Tanabe, Kyoto University (Japan)

John M. Zavada, Polytechnic Institute of New York University (United States)

Jun Zhang, U.S. Army Research Laboratory (United States) 


\section{Session Chairs}

1 Rare-Earth Doped Materials I

Shibin Jiang, AdValue Photonics, Inc. (United States)

2 Metamaterials

Michel J. F. Digonnet, Stanford University (United States)

3 Silicon Photonics

Shibin Jiang, AdValue Photonics, Inc. (United States)

4 Sensors

Rolindes Balda, Universidad del País Vasco (Spain)

5 Gratings

Jun Zhang, U.S. Army Research Laboratory (United States)

6 Modulators and Filters

Jesse A. Frantz, U.S. Naval Research Laboratory (United States)

$7 \quad$ Nanomaterials

Angel Flores, Air Force Research Laboratory (United States)

8 Optical Glasses, Fibers, and Waveguides

Leonid B. Glebov, CREOL, The College of Optics and Photonics, University of Central Florida (United States)

9 Rare-Earth Doped Materials II

Jian Xu, Kyoto University (Japan)

10 Detectors

Joel Bagwell, Edmund Optics Inc. (United States)

11 Development of Optical Components

Michel J. F. Digonnet, Stanford University (United States) 